\title{
New Algorithm for Generating a Very Long Rectangular Turbulent Phase Screen
}

\author{
Han-Ling $\mathrm{Wu}^{\mathrm{a}}$, Hai-Xing Yan ${ }^{\mathrm{a} 1}$, Shu-Shan $\mathrm{Li}^{\mathrm{a}}$ and Xin-Yang $\mathrm{Li}^{\mathrm{b}}$ \\ ${ }^{a}$ Institute of Mechanics, Chinese Academy of Sciences, Beijing 100190, P.R. China \\ ${ }^{\mathrm{b}}$ Institute of Optics and Electronics, Chinese Academy of Sciences, Chengdu 610209, P.R. China
}

Based on the fractal characteristics of turbulence-distorted wavefront, a new algorithm for generating a very long rectangular turbulent phase screen is proposed. The phase structure function of generated phase screens can be very well compared to the theoretical one. In comparison to existing approaches, the present algorithm shows obvious advantages.

Generally, the multiple phase screen method is used to do numerical simulations of laser propagation through a turbulent media and an adaptive optics system for phase compensation ${ }^{[1]}$. Phase screens are used to describe the turbulent media such as a turbulent atmosphere. The structure constant of the index of refraction fluctuations, $C_{n}^{2}$, is used to characterize the turbulence strength. The main idea of the multiple phase screen method is as following. The propagation path through the turbulent media can be divided into several segments which may have different lengths. It is thought that each segment may deform the phase of the optical wave independently. The contribution of the turbulent media segment to the phase distortion of the laser beam can be "pressed" into a very thin phase screen and added to the original phase of the optical wave. It is assumed that the phase screen does not have a significant influence on the amplitude of the wave. The amplitude of the optical wave varies only in the propagation process of the wave having the deformed phase between two successive phase screens.

Obviously, the generation and application of turbulent phase screens which characterize the turbulence is a key problem of the multiple phase screen method. In practical application of turbulent phase screens, for example, in numerical simulation of laser propagation through the atmosphere with the phase compensation by an adaptive optics system including temporal evolution, such as very long exposure imaging and simulation of the dynamic control process in an adaptive optics system ${ }^{[2]}$, very long even infinitely-long rectangular phase screens must be utilized. But, the widely-used spectral approach can only generate square phase screen and wastes the computational resource because only a rectangular portion of the square phase screen is practically utilized. Furthermore, the spectral phase screen needs the low frequency modification, so that it needs more amount of computations.

Based on the fractal characteristics of turbulence-distorted wavefront, a new algorithm for generating very long even infinitely-long rectangular turbulent phase screen is proposed in this paper. The phase structure function of generated phase screens can be very well compared to the theoretical one. In comparison to existing approaches, the present algorithm shows obvious advantages: simplicity, high efficiency and less computer resource. Furthermore, the generated phase screen shows better agreement with theoretical result in the turbulent statistical characteristics, especially, in the low frequency region and the high frequency region.

[1] Hai-Xing Yan, Shu-Shan Li, De-Liang Zhang, She Chen, "Numerical simulation of an adaptive optics system with laser propagation in the atmosphere,” Appl. Opt. 39(18), 3023-3031 (2000)

[2] Hai-Xing Yan, Shu-Shan Li, She Chen, "Numerical simulation investigations of the dynamic control process and frequency response characteristics in an adaptive optics system," Proc. SPIE 4494, 156-166 (2002)

\footnotetext{
${ }^{1}$ E-mail: hxyan@imech.ac.cn
} 